\title{
Faut-il autoriser des aides d'exploitation pérennes versées par les aéroports régionaux aux compagnies à bas coûts?
}

\author{
Estelle Malavolti* \\ Frédéric Marty
}

\begin{abstract}
Résumé
Les lignes directrices sur les aides publiques, modifiées en 2014, ouvrent désormais la possibilité d'aides à l'exploitation. Nous analysons économiquement ces mesures de soutien, pour montrer qu'elles peuvent s'avérer rationnelles pour le gestionnaire d'une infrastructure aéroportuaire, et donc être compatibles avec le critère de l'investisseur privé en économie de marché. A cette fin, nous proposons une modélisation de l'aéroport comme une plateforme biface, exploitant les externalités présentes entre activités aéronautiques et commerciales. Nous montrons en outre qu'un lien existe entre l'intensité de l'aide et le mode de régulation ex ante des redevances aéroportuaires. Si ces dernières sont régulées par prix plafonds, la compagnie aérienne dont le pouvoir de négociation est plus élevé pourra s'approprier la plus large part des gains de l'échange.
\end{abstract}

\section{State aids to low-cost airlines : could it be worth if durable?}

\section{Résumé}

The guidelines on state aids, issued in 2014, provide simpler start-up aids rules for new routes and open the way to transitory operating aid schemes. These measures as discounts on landing or on terminal charges, can make sense at the economic point of view and can comply with criterion of the private market investor principle. For this purpose, we model the airport as a two-sided platform, performing a trade-off between its aeronautical and commercial resources. Furthermore, we put into relief the relationship between the level of the subsidy and the airport's charges regulation. If regulation uses a price-cap mechanism, the airline may abuse of its negotiation power to extract most of the contract's gains. Conversely, a regulation based on a floor mechanism may allow to limit the capacity of the airline to extract gains and to reduce by way the level of the subsidy.

Mots Clés : Aides d'État, marché biface, transport aérien, redevances aéroportuaires, state aids, two-sided market, air transport, aeronautical charges.

JEL codes : D43, K23, L13, L43, L93.

${ }^{*}$ University of Toulouse, ENAC and TSE, 7 avenue Ed. Belin, pièce Z132, CS54005, 31500 Toulouse cedex 4, estelle.malavolti@tse-fr.eu

${ }^{\dagger}$ CNRS, GREDEG, Université Nice Sophia Antipolis, 250 rue A. Einstein, 06560 Valbonne, frederic.marty@gredeg.cnrs.fr.

${ }^{\ddagger}$ Les auteurs remercient les rapporteurs pour leurs commentaires et conseils. 


\section{Introduction}

Tant les autorités en charge de l'application des règles de concurrence que les autorités de contrôle des comptes publics ont fréquemment à se pencher sur la situation des aéroports secondaires et sur les conventions que peuvent nouer des compagnies à bas coûts (low cost carriers ci-après LCCs). Certains accords sont dénoncés comme excessivement favorables aux compagnies en question. Ils posent à ce titre deux types de problèmes.

Un premier problème tient aux effets concurrentiels de ces accords. Les soutiens dont peuvent bénéficier les LCCs peuvent être considérés comme des aides publiques. Celles-ci devraient être notifiées afin de s'assurer de leur compatibilité avec les règles de fonctionnement du marché intérieur. Dans le cadre de l'approche plus économique mise en œuvre par la Commission, une aide publique peut être autorisée si elle répond à une défaillance de marché identifiée. Les aides ne sont donc pas interdites en elles-mêmes mais elles doivent être préalablement notifiées et ne pas induire de distorsions de concurrence entre compagnies aériennes. La Commission européenne a été à l'origine de nombreuses procédures formelles depuis le début des années 2000 conduisant à l'annulation de nombreuses conventions. Celles-ci se traduisent par une requalification des éventuelles mesures de soutien en aides publiques et une injonction par la LCC de remboursement des fonds versés par le gestionnaire de l'infrastructure aéroportuaire ${ }^{1}$.

Un second problème tient aux effets budgétaires de ces accords. En effet, les aéroports secondaires européens se caractérisent par des déficits d'exploitation souvent liés à l'existence de surcapacités structurelles. Les soutiens à des compagnies aériennes pour générer du trafic peuvent effectivement limiter un éventuel déficit en permettant de couvrir les coûts variables et d'amortir au moins une partie des coûts fixes. Ce soutien peut participer de la rationalité qui serait celle d'un investisseur privé en économie de marché. Un gestionnaire d'infrastructure peut rationnellement soutenir par des réductions de redevances aéronautiques une LCC, et ce, de façon pérenne ${ }^{2}$. En revanche, la question de la répartition des gains relatifs peut être posée. En effet, le contrat entre la compagnie aérienne et le gestionnaire de l'infrastructure aéroportuaire ne met pas en lien deux agents dotés du même pouvoir de négociation. La LCC peut obtenir de fortes réductions de redevances aéroportuaires pouvant laisser craindre des distorsions de concurrence entre compagnies (Malavolti et Marty [2010]), mais aussi des phénomènes collectivement sous-optimaux de concurrence fiscale (Malina [2012]). La difficulté vient principalement du fait que les aéroports secondaires présentent des sur-capacités et que les possibilités d'arbitrage des LCC entre les différentes destinations les placent en situation de monopsone ${ }^{3}$. Les conventions signées par

1. Ce fût le cas par exemple à Nîmes pour Ryanair (SA.22961, 6.4 Million $€$ ), à Pau pour Ryanair (SA.22614, 2.4 Million $€$ ) et Transavia (400000 €).

2. La Commission Européenne a pris position dans certains cas en faveur du principe d'investisseur privé en économie de marché. C'est le cas pour Frankfurt Hahn et Ryanair (IP/08/956), Saarbrücken Airport et Air Berlin (IP/12/156).

3. Starkie, dans le cadre du rapport OCDE sur l'analyse des interactions stratégiques entre compagnies aériennes et aéroports [2009] précise en effet que les aéroports peuvent plus difficilement exploiter leur pouvoir de marché du moment que l'aéroport est en sur-capacité et que la concurrence directe sur l'aéroport entre compagnies 
les aéroports et compagnies peuvent refléter ce déséquilibre. Par exemple, le Conseil Supérieur de l'Aviation Civile [2017] note l'existence de contrats marketing portant non seulement sur une baisse des redevances aéronautiques mais également sur un partage des recettes commerciales de l'aéroport ${ }^{4}$.

L'apport de notre travail est de proposer une modélisation en marché biface permettant de réaliser une analyse économique du critère d'investisseur privé en économie de marché.

Les revenus de l'aéroport ont en effet deux origines : le produit des redevances aéronautiques d'une part, et les recettes commerciales (parkings, loyers des sous-concessions des espaces commerciaux...) d'autre part. Ce modèle a été développé pour les plates-formes d'intermédiation (Rochet et Tirole [2003], [2006], Armstrong [2006], Hagiu et Wright [2015], Verdier [2016]). Une plate-forme biface se caractérise par les effets externes de réseau qu'elle génère entre les participants. La présence de consommateurs sur l'un des versants de la plate-forme crée de la valeur sur l'autre versant, faisant qu'il peut être optimal de distordre la structure tarifaire entre les deux versants pour maximiser les revenus. Les externalités peuvent jouer à double sens ou simplement d'un sens à l'autre. Nous appliquons ce cadre théorique aux infrastructures aéroportuaires suivant en ceci une part croissante de la littérature.

Les premières analyses des aéroports en termes de marchés biface ont été développées par Gillen [2011], Malavolti [2014], Ivaldi et al. [2015]. Gillen [2011] mit en évidence la possibilité de générer des revenus commerciaux additionnels pouvant compenser des réductions sur les tarifs aériens permettant aux compagnies d'accroître leurs dessertes et donc le nombre de passagers utilisant l'infrastructure. Le poids des revenus non aéronautiques dans l'équilibre économique des aéroports ne saurait être négligé. Par exemple, en 2014, plus de 60\% des profits de Aéroports de Paris sont de source commerciale. Ce pourcentage n'a fait que croître entre 2009 (54\%) et 2014 (61\%) (ADP, [2014]). Les premières analyses empiriques aux États-Unis réalisées par Ivaldi et al. [2015] montrent l'existence des externalités entre les activités commerciale et aéronautique, justifiant une approche biface de l'aéroport.

C'est pour cette raison que nous préférons privilégier l'approche biface à celle en termes de chaîne verticale pour formaliser le modèle d'affaire de l'aéroport. Les critiques formulées à l'encontre de cette approche pour les aéroports tiennent principalement au fait que les externalités ne sont observées que d'un versant sur l'autre et non de façon croisée (voir par exemple, Fröhlich [2011]). Cependant, les récents développements de l'analyse biface étendent la validité du modèle

aériennes est faible. Par ailleurs, le Conseil Supérieur de l'Aviation Civile dans un rapport de 2017 sur le maillage aéroportuaire français estime notamment que les relations entre les compagnies et les aéroports à faible activité sont caractérisées par des "monopoles inversés" de la part des compagnies aériennes.

4. Ces contrats peuvent être assimilés selon le CSAC aux marges arrières imposées par la grande distribution aux petits producteurs. En cela, ils posent nécessairement des questions de restrictions verticales qui ne seront pas directement abordés dans notre article pour le moment. Voire par exemple Rey [2003] pour une analyse économique générale des restrictions verticales et Wright [2007] pour une analyse de leur impact sur le bien-être des consommateurs. On peut noter que les accords de partage commerciaux dans l'aérien peuvent avoir des effets bénéfiques comme montré par Fu et Zhang [2010], car ils internalisent des effets de demande. Ils nuisent néanmoins à la concurrence entre compagnies. 
au cas d'externalité unidirectionnelle (Hagiu et Wright [2015]). Le modèle reste donc valide même si la décision de voyage des passagers n'est pas univoquement affectée par l'offre de services commerciaux de l'infrastructure aéroportuaire.

Notre modèle propose de répondre également à des problèmes de régulation sectorielle. Il s'agit de s'interroger sur l'impact des réductions de redevances sur l'équilibre économique de l'infrastructure aéroportuaire. En effet, les redevances aéroportuaires font l'objet en Europe d'une régulation par prix plafond ${ }^{5}$. Les plates-formes secondaires n'arrivent que très rarement à équilibrer leurs comptes (ECA [2014]). Le problème tient au fait que ce déficit va être compensé par des fonds publics. Il s'agit donc de concilier le comportement optimal de l'opérateur de l'infrastructure avec la minimisation de son déficit. Notre modèle montre que le mode de régulation ex ante des redevances aéronautiques n'est pas neutre en termes de répartition des gains de l'échange pour les deux parties à la convention. Si une régulation par prix plafond garantit qu'un aéroport en situation de monopole n'extorque pas une part excessive de la rente des compagnies aériennes, elle ne peut limiter l'exercice du pouvoir de marché d'une compagnie en situation de monopsone.

En effet, si la nature biface du marché rend possible la compensation du manque à gagner sur le versant aéronautique par des recettes additionnelles sur le versant commercial, on peut même envisager une exonération complète de redevance, voire une redevance négative ${ }^{6}$. Cela peut par exemple prendre la forme d'un partage des revenus commerciaux avec la LCC. Pour autant, si un gain net par rapport à la situation initiale résulte de cet accord pour l'ensemble des parties prenantes, on ne peut pas présumer de sa répartition entre LCC et gestionnaire.

Par ailleurs, la réduction des redevances est compensée par des ressources publiques. Une aide publique est en jeu qu'elle aille directement vers la LCC ou qu'elle vienne soutenir l'équilibre d'exploitation de l'infrastructure aéroportuaire. Il existe donc une interdépendance entre la réglementation ex ante des redevances aéronautiques et le contrôle ex post de l'intensité de l'aide publique par le juge de la concurrence. Ainsi, nous visons, dans le cadre de cet article, à montrer de quelle façon les modalités de réglementation ex ante (par prix plafond ou prix plancher) peuvent jouer sur les intensités des aides telles qu' évaluées ex post mais aussi sur la répartition des gains du contrat entre les différents acteurs.

Dans les faits, la conformité de la mesure de soutien à l'encadrement européen des aides publiques est évaluée ex post si le critère de l'investisseur privé en économie de marché est en jeu ou, ex ante, par voie de notification s'il s'agit d'une aide publique. Nous supposons, dans notre modèle, que la mesure de soutien est un taux de réduction sur les redevances ${ }^{7}$. Ainsi, tout se

5. Cette régulation vise à prévenir que le gestionnaire n'abuse de son pouvoir de marché, issu a priori de sa situation de monopole naturel. Cependant, les aéroports secondaires ne jouissent pas d'un tel pouvoir de marché dans la mesure où ils doivent rentabiliser des infrastructures existantes, pérenniser des dessertes dans un contexte dans lequel les compagnies aériennes peuvent aisément arbitrer entre plusieurs aéroports et représentent l'unique client de l'infrastructure.

6. Nous avons montré par ailleurs que la gratuité sur un versant d'une plateforme biface peut être rationnelle économiquement (voir Malavolti et Marty [2013]).

7. D'autres mesures de soutien existent, telles les co-financement de campagnes promotionnelles, que nous 
passe comme si la LCC négociait de facto une subvention globale avec l'aéroport. C'est la raison pour laquelle nous simplifions l'analyse en considérant que le contrôle de l'aide porte sur une subvention "forfaitaire".

Notre travail permet également de comprendre le lien entre la réglementation des redevances ex ante et le contrôle ex post des aides publiques. Il montre qu'un moyen de limiter l'intensité des aides (et donc les capacités d'appropriation du surplus par les LCC) pourrait être l'instauration d'une régulation des redevances par plancher et non plus par plafond. En d'autres termes, une régulation par prix plancher des redevances aéronautiques dans les aéroports dépourvus de pouvoir de marché permet de limiter la part du surplus créé par la convention que peut s'approprier la LCC. Nous ne réalisons pas une analyse en termes de bien-être mais l'idée sous-jacente est que plus forte est la part de surplus que s'approprie la LCC, plus important sera le soutien nécessaire à l'équilibre d'exploitation de l'aéroport secondaire; soutien passant par des injections de fonds publics coûteux en termes collectifs.

Nous présentons dans la prochaine section le modèle et ses principaux résultats. Notre dernière section discute les résultats obtenus et propose des pistes de recherches futures.

\section{Modélisation des aides d'État à destination des aéroports se- condaires}

Notre modèle considère l'aéroport comme une plate-forme qui et en relation des passagers avec les magasins se situant dans le terminal. Cette interaction se fait par l'intermédiaire des compagnies qui amènent le trafic sur l'aéroport. Vont ainsi être fixées une redevance aéronautique $a$ dépendant du nombre de passagers voyageant avec la compagnie aérienne et un prix $r$ pour l'espace commercial loué. La demande de transport exprimée en nombre de passagers, est fonction du prix du ticket. On note $N(p)$, la demande de voyage adressée aux compagnies aériennes, où $p$ est le prix du ticket payé par chaque passager. Cette demande est décroissante avec le prix du ticket et maximale pour un prix du ticket nul. Elle vaut dans ce cas $\bar{N}$. La redevance aéroportuaire est proportionnelle par principe de calcul à cette demande. en outre, les activités non aéronautiques sont majoritairement représentées par la demande de location d'espaces à l'intérieur du terminal (pour les magasins) ou à l'extérieur du terminal (pour les loueurs de voitures). Cette demande, notée $S(r, N)$, où $S$ représente le nombre d'emplacements, est fonction décroissante du prix de location $r$, et croissante du nombre de passagers $N$, car ils représentent les clients potentiels pour ces magasins ou loueurs ${ }^{8}$. On suppose par ailleurs qu'il n'y a pas

assimilons par commodité à cette modalité dans notre analyse.

8. Nous faisons le choix d'une externalité positive exercée sur les revenus des magasins par les flux de passagers. Certains modèles bifaces considèrent en effet des externalités croisées entre les deux côtés du marché (jeux vidéo, centres commerciaux, journaux...). Dans le cas présent il est en effet possible de considérer que les magasins exercent également une externalité sur les passagers. Cependant, le signe de cette externalité est difficile à déterminer : on peut envisager que la présence de magasins est souhaitée par les passagers. Attendre dans un aéroport entouré de magasins est sans doute préféré par les consommateurs. Mais certaines études ont montré 
d'effets croisés du prix de la location et du nombre de clients.

Les coûts des aéroports sont composés de coûts variables, essentiellement dépendants du nombre de passagers présents dans l'aérogare et notés $C V(N)$, fonction croissante de $N$; et de coûts fixes, notés $C F$, correspondants aux investissements réalisés (piste, aérogare, parkings, locaux commerciaux...) afin d'installer une capacité de production des services aéronautiques et non aéronautiques. Ainsi, le profit de la structure aéroportuaire peut s'écrire :

$$
\Pi_{\text {aéroport }}=a N(p)-C V(N(p))+r S(r, N(p))-C F
$$

Où $a N(p)$ représente les recettes aéronautiques, $r S(r, N(p))$, les recettes commerciales, $C V(N(p))$ $+C F$, les coûts totaux de l'aéroport. Les aéroports viables financièrement sont surtout les aéroports à forte activité (Aéroports de Paris - ADP, Aéroport de Francfort - Fraport...). Ce ne sont donc par définition pas les aéroports qui sont concernés par les aides publiques de type investissement. Les plate-formes aéroportuaires qui nous intéressent sont celles qui, au vu de la faiblesse de leur demande de services (passagers et magasins) combinée avec des coûts fixes élevés, ne sont pas rentables. Les aides publiques se justifient dès lors que le profit variable est positif, i.e. si on se situe dans une zone où les coûts variables sont couverts, mais pas les coûts fixes en totalité. L'hypothèse technique qui correspond à cette situation est alors la suivante : le profit de la structure est supposée négatif même dans le meilleur cas de figure possible, i.e. pour toutes valeurs et combinaisons de $N(p)$, de $S$, de $r$ et de $a$, le profit total est négatif sans aide supplémentaire. Un montant fixe, noté $A$ dans notre modèle, est alors versé à la structure. Ce montant est indirectement limité par l'Autorité de Concurrence en compatibilité avec ses lignes directrices et sa pratique décisionnelle. La LCC va donc déduire de celles-ci quel sera le montant maximal de l'aide qui pourra être accepté, montant noté $\bar{A}$.

En outre, la redevance aéronautique payée par la compagnie est régulée sur la base d'une situation théorique de monopole naturel. Le régulateur fixe un prix plafond, noté $\bar{a}^{9}$, en considérant l'ensemble des recettes et coûts de l'aéroport.

que les temps d'attente pouvait être rallongés du fait de la présence de magasins, prêts à payer plus cher leur location pour avoir plus longtemps les passagers à l'aéroport. Voir par exemple Torres et al., 2005 et Malavolti, 2014. Les études n'étant pas concluantes quant au signe, nous préférons ne pas considérer d'externalité exercée par les magasins sur les passagers ex ante. En revanche, les résultats seront discutés en fonction du signe de cette externalité.

9. Le périmètre de réglementation est différent selon la taille des aéroports : les petits aéroports européens, comme recommandé par l'OACI (document 9082), sont plutôt régulés en caisse unique alors que les plus grosses structures optent pour une régulation en double caisse. La caisse unique correspond à une situation dans laquelle le périmètre de régulation inclut toutes les recettes de l'aéroport. Le principe de double caisse correspond à une situation dans laquelle seules les recettes aéronautiques sont incluses. La littérature économique s'est intéressée à ce problème, avec des conclusions différentes. Une première série de papiers (Starkie [2001]; Starkie et Yarrow [2008]) analyse l'impact du type de régulation sur les objectifs à long terme de l'aéroport. Les articles montrent, en utilisant une approche de coût du capital, que les incitations à investir de l'aéroport sont réduites s'il n'est pas en mesure de conserver suffisamment de ressources. Ils concluent donc qu'une régulation en double caisse est préférable. Cependant, des articles plus récents (Fröhlich [2011]; Malina et al. [2012]; Malavolti [2014]) obtiennent des conclusions opposées dès lors que l'aéroport est considéré comme une plate-forme. Ces travaux recommandent une régulation en caisse unique de façon à tenir compte des externalités existantes entre les deux côtés du marché, aéronautique et commercial. Enfin, Perrot [2014] suggère que, dans le cas de gros aéroports, les problèmes de 
Dans notre analyse, l'aéroport a une seule compagnie aérienne comme cliente, dont il est considéré comme étant économiquement dépendant. La compagnie aérienne va faire une offre à prendre ou à laisser ${ }^{10}$ à l'aéroport sous la forme d'un contrat fixant $(a, r, A)$, tout en s'assurant que l'aéroport ait intérêt à accepter cette offre. Dans le cas de petits aéroports secondaires, l'activité dépend d'un contrat fourni par une seule compagnie. On va donc considérer que l'aéroport va accepter de participer tant que le profit résultant de l'accord est non négatif ${ }^{11}$. En effet, on peut prendre en compte le fait que certains coûts fixes de l'infrastructure sont irrécupérables et qu'ils devront être payés que la compagnie propose un contrat ou non à l'aéroport. Il s'agit par exemple de tenir compte des coûts fixes des installations en place (piste, terminal). En revanche, une partie des coûts fixes ne sera engagée que dès lors que le contrat sera accepté, comme par exemple les coûts fixes inhérents à l'organisation des espaces commerciaux ou la réalisation/mise aux normes d'un parking extérieur. Par simplicité, nous normalisons les coûts fixes irrécupérables à zéro.

L'objectif de notre article est de proposer une modélisation de la relation entre une LCC et un aéroport économiquement dépendant, et de comprendre comment les outils réglementaires ex ante (la redevance aéronautique maximum) et ex post (l'aide à l'activité) interagissent et influencent les décisions des acteurs économiques. Le jeu que nous nous proposons d'étudier a la forme suivante représentée dans la figure 1.

congestion peuvent justifier une régulation en double caisse. En effet, la conséquence sur la structure de prix d'équilibre des externalités positives exercées par les passagers sur les magasins est que les magasins subventionnent les ristournes sur les taxes aéroportuaires et donc indirectement les passagers. A l'équilibre, plus de passagers sont donc présents à l'aéroport, ce qui accroît les problèmes de congestion. Ainsi, si les coûts sociaux de la congestion sont suffisamment importants, il peut être préférable de réguler l'aéroport en deux caisses séparées.

10. À titre d'illustration, on peut noter que certains aéroports concluent des contrats de partage de leur profit commercial. C'est le cas par exemple de Ryanair en France qui conditionne le lancement de ses services dans certains aéroports au partage des recettes de parking. C'est le cas également de Tampa en Floride où l'aéroport partage les recettes des concessions commerciales avec les compagnies opérant sur ses infrastructures. Fu et al. [2011] présentent cet exemple dans leur article sur l'analyse des relations verticales entre compagnies aériennes et aéroports.

11. Le profit que l'aéroport peut réaliser en dehors de la transaction peut également correspondre à la possibilité de conclure un contrat avec une autre compagnie. À cette condition, l'aéroport choisira entre les différents contrats qu'on lui propose, le plus avantageux. Cependant, ces contrats peuvent être incertains ou même inexistants. Dans tous les cas, les aides publiques concernées par ces contrats relèvent plutôt des aides au démarrage d'activité, concernant des nouvelles lignes ouvertes. Nous nous focalisons sur les aides à l'activité éventuellement pérennes. 
Figure 1 - Arbre de décision

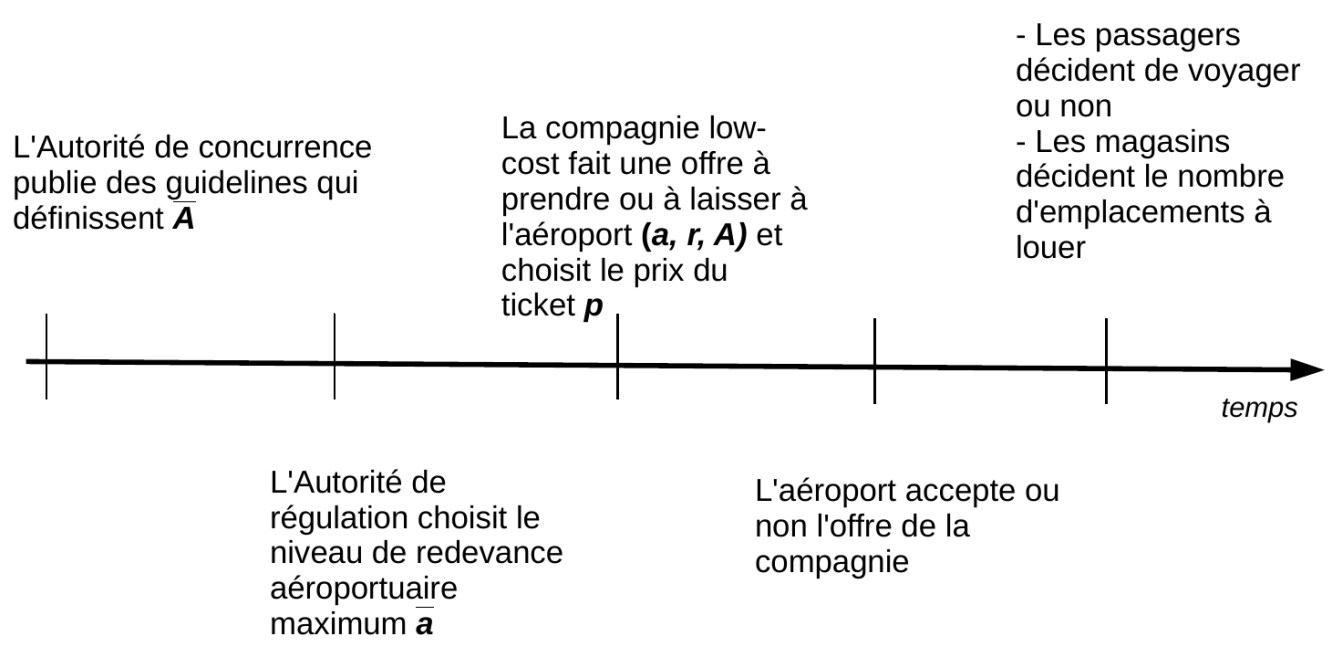

Le programme de la compagnie aérienne s'écrit de la manière suivante :

$$
\begin{array}{cl}
\underset{\{p, r, a, A\}}{\operatorname{Max} \Pi_{\mathrm{LCC}}=} & p N(p)-a N(p)-C(N(p)) \\
\text { s.t. } & \Pi_{\text {aéroport }}=a N(p)-C V(N(p))-C F+A+r S(r, N(p)) \\
\quad a \leq \bar{a} & \geq 0 \\
\quad 0 \leq A \leq \bar{A} & \left(C_{1}\right) \\
& p \in \mathbb{R}^{+}, r \in \mathbb{R}^{+}, a \in \mathbb{R}
\end{array}
$$

$p N(p)$ représente les recettes de la compagnie tirées de la vente de $N$ tickets d'avion au prix $p . a N(p)$ représente les coûts d'accès aux services aéronautiques payés par la compagnie à l'aéroport pour prendre en charge $N$ passagers. $C(N(p))$ correspond à l'ensemble des coûts variables supportés par la compagnie lorsqu'elle fait voyager $N$ passagers ${ }^{12}$. Cette fonction est croissante et convexe en $N$. Les contraintes $C_{2}$ et $C_{3}$ représentent les contraintes réglementaires à satisfaire. Le niveau de redevance aéronautique retenu $a$ ne peut en effet pas dépasser le prix plafond imposé par le régulateur $\bar{a}$. Rien n'empêche en revanche la redevance aéronautique d'être l'équivalent d'une subvention, i.e. $a^{*}<0$ à l'équilibre si l'optimisation du profit l'y conduit. L'aide d'activité $A$ demandée est quant à elle nécessairement positive et ne peut excéder le niveau maximal autorisé par les autorités de concurrence. La contrainte $C_{1}$ représente la contrainte de participation de l'aéroport.

Proposition 1 À l'équilibre, la LCC extrait tout le profit réalisé par l'aéroport à l'aide notamment de l'aide d'activité demandée, qui est maximale.

$$
\Pi_{\text {aéroport }}=0
$$

12. Nous normalisons les coûts fixes de la compagnie aérienne à zéro par souci de simplification. 


$$
A^{*}=\bar{A}
$$

\section{Preuve.}

Soit le quadruplet solution du programme de maximization $\left(p^{*}, r^{*}, a^{*}, A^{*}\right)$ tel que $C_{1}\left(p^{*}, r^{*}, a^{*}, A^{*}\right)>0$. Alors il existe $\tilde{a}<a^{*}$ tel que $C_{1}\left(p^{*}, r^{*}, \tilde{a}, A^{*}\right)>0$ et tel que $\tilde{a}$ satisfait les autres contraintes $C_{2}$ et $C_{3}$. Le profit de la compagnie est alors supérieur puisque seul $a$ est modifié. $\Pi_{\mathrm{LCC}}\left(p^{*}, r^{*}, \tilde{a}, A^{*}\right)>\Pi_{\mathrm{LCC}}\left(p^{*}, r^{*}, a^{*}, A^{*}\right)$. Cette solution est donc préférable. Il n'est donc pas possible de trouver une solution telle que la contrainte $C_{1}$ ne soit pas saturée à l'optimum.

Supposons par ailleurs que $A^{*}<\bar{A}$, alors $C_{1}\left(p^{*}, r^{*}, a^{*}, \bar{A}\right)>C_{1}\left(p^{*}, r^{*}, a^{*}, A^{*}\right) \geq 0$. Il est alors possible de trouver $\tilde{a}<a^{*}$ tel que $C_{1}\left(p^{*}, r^{*}, \tilde{a}, \bar{A}\right)=C_{1}\left(p^{*}, r^{*}, a^{*}, A^{*}\right) \geq 0$ qui apporte un profit supérieur à la compagnie aérienne. $\Pi_{\mathrm{LCC}}\left(p^{*}, r^{*}, \tilde{a}, \bar{A}\right)>\Pi_{\mathrm{LCC}}\left(p^{*}, r^{*}, a^{*}, A^{*}\right)$. En conclusion, la contrainte $C_{3}$ est saturée à l'optimum.

Ce résultat est robuste car non dépendant de la forme des fonctions de profits de la compagnie aérienne et de l'aéroport, mais de conditions naturelles nécessaires à la convexité du problème, nous indique que la LCC possède suffisamment de variables de décisions pour pouvoir extraire tout le profit auprès de l'aéroport et ce notamment grâce à l'aide à l'activité à laquelle l'aéroport peut avoir accès. Ce résultat met, surtout et de manière plus intéressante, en avant l'interaction entre les instruments de régulation ex ante, i.e. la redevance aéronautique maximale, et ex post, l'aide à l'activité maximale qui peuvent être demandées. Plus précisément, il apparaît que la redevance aéronautique et l'aide publique demandées sont des instruments substituts pour la compagnie aérienne pour satisfaire la contrainte de participation de l'aéroport. En effet, plus l'aide d'activité est élevée et plus la contrainte de participation est facile à satisfaire, alors que le profit de la compagnie ne s'en trouve pas modifié. De sorte que la compagnie aérienne, sensible au niveau de la redevance aéronautique puisqu'elle représente un coût direct, peut utiliser son pouvoir de marché pour baisser la redevance et ainsi augmenter son profit, sans pour autant modifier les contraintes. L'aide publique sera donc fixée à son maximum. La contrainte de participation de l'aéroport sera elle aussi saturée car, sans modification des autres arguments de la maximisation, elle peut être ajustée par le niveau de la redevance aéronautique. Celle-ci sera fixée au niveau le plus faible possible de façon à satisfaire la contrainte de participation de l'aéroport. Ainsi, il se peut tout à fait que la redevance optimale du programme soit en définitive une subvention que l'aéroport verse à la compagnie aérienne pour attirer des passagers, futurs clients des magasins de l'aéroport. La structure biface prend ici tout son sens : cette solution n'est envisageable que dans la mesure où la partie variable du profit de l'aéroport est suffisamment important par rapport à $-C F+\bar{A}$. Cette partie variable est composée des recettes aéronautiques et commerciales. Si les recettes commerciales sont suffisantes, la compagnie aérienne pourra alors réduire d'autant la redevance aéronautique pour augmenter son profit.

Pour des raisons de clarté, nous modifions le programme de façon à optimiser par rapport au nombre de passagers $N$. Ceci est possible car la fonction $N(p)$ est décroissante strictement en $p$ par définition. Par ailleurs, suite à la Proposition 1, la contrainte de participation de l'aéroport permet de fixer le niveau optimal de la redevance aéronautique. Il suffit alors de la réinjecter dans la contrainte de régulation de la redevance aéronautique $C_{2}$ pour avoir le programme transformé suivant : 


$$
\begin{array}{cc}
\underset{\{N, r\}}{\operatorname{Max} \Pi_{\mathrm{LCC}}}= & p(N) N+r S(r, N)+\bar{A}-C V(N)-C F-C(N) \\
\text { s.t. } & C V(N)+C F-\bar{A}-r S(r, N)-\bar{a} N \leq 0 \\
& N \in \mathbb{R}^{+}, r \in \mathbb{R}^{+}
\end{array}
$$

La fonction objectif de la compagnie est composée non seulement du profit réalisé après la vente des billets d'avion aux passagers $(p(N) N-C(N))$, mais également du profit de l'aéroport amputé de la redevance aéronautique puisqu'elle était un coût pour la compagnie $(r S(r, N)-$ $C V(N)-C F)$. La compagnie intègre le fait qu'elle demandera le maximum disponible d'aide publique pour faciliter l'acceptation par l'aéroport de l'offre qu'elle va lui faire.

Soit $\mu \in \mathbb{R}^{+}$le multiplicateur de Lagrange associé à la contrainte $\left(C_{1}\right)$ du programme de maximisation de la compagnie aérienne. Le programme devient un programme de maximisation sous la forme d'un Lagrangien $\mathcal{L}(r, N)$ dont les conditions de premier ordre s'écrivent :

$$
\begin{aligned}
& \frac{\partial \mathcal{L}}{\partial N}=p\left(N^{*}\right)+N^{*} \frac{\partial p}{\partial N}-\frac{\partial C}{\partial N}+\left(1+\mu^{*}\right)\left(r^{*} \frac{\partial S}{\partial N}\right)-\left(1+\mu^{*}\right) \frac{\partial C V}{\partial N} \quad-\mu^{*} \bar{a}=0 \quad(C N 1) \\
& \frac{\partial \mathcal{L}}{\partial r}=\left(1+\mu^{*}\right)\left(r^{*} \frac{\partial S}{\partial r}+S\left(r^{*}, N^{*}\right)\right) \quad=0 \quad(C N 2) \\
& \mu^{*}\left(C V\left(N^{*}\right)+C F-\bar{A}-r^{*} S\left(r^{*}, N^{*}\right)-\bar{a} N^{*}\right) \quad=0 \quad(C N 3),
\end{aligned}
$$

La condition $(C N 2)$ revient à fixer le prix de location des espaces commerciaux au niveau du prix de monopole. En effet, pour tout $\mu \geq 0,(C N 2)$ est vrai ssi $r^{*} \frac{\partial S}{\partial r}+S\left(r^{*}, N^{*}\right)=0$. La compagnie se comporte en monopole, comme l'aurait fait l'aéroport lui-même, vis-à-vis des magasins. On retrouve ainsi le résultat classique de l'application d'un taux de marge par la compagnie par rapport aux coûts marginaux (ici nuls dans notre modèle), taux de marge qui est d'autant plus important que la sensibilité de la demande d'espaces commerciaux par rapport au prix de la location est faible, i.e. $\frac{\partial S}{\partial r}$ est faible. En outre, plus $N^{*}$ sera élevé et plus $r^{*}$ le sera également, puisque la demande d'espaces commerciaux est croissante avec le nombre de passagers dans l'aéroport, conséquence de la prise en compte dans notre modèle biface de l'externalité exercée par l'activité aéronautique sur l'activité commerciale ${ }^{13}$.

La première condition $(C N 1)$ est composé d'un premier élément $p\left(N^{*}\right)+N^{*} \frac{\partial p}{\partial N}$ correspondant à la condition de maximisation du profit de la compagnie aérienne si elle n'était pas en position dominante. Cette condition est celle qui fixerait le prix de monopole pour les tickets vendus aux passagers. Plusieurs effets additionnels sont pris en compte, notamment les coûts marginaux de l'aéroport $\frac{\partial C V}{\partial N}$ qui vont avoir tendance à diminuer le nombre de passagers optimal, la prise en compte du profit commercial de l'aéroport qui est croissant en le nombre de passagers, $\left(r^{*} \frac{\partial S}{\partial N}\right)$,

13. À noter que la solution $r^{*}$ aurait été la même si elle avait été choisie librement par l'aéroport considérant les deux faces du marché, puisqu'il aurait appliqué son pouvoir de monopole et déterminé ainsi le même niveau de prix de location d'espace. Voir Malavolti [2014] pour une analyse de l'impact de la nature biface du marché sur la structure des prix à l'équilibre. 
et enfin la prise en compte de la contrainte de régulation de la redevance aéronautique qui va dans le sens de diminuer le nombre de passagers à l'équilibre si la contrainte n'est pas saturée (plus exactement si le multiplicateur n'est pas nul à l'équilibre, i.e. $\mu^{*}>0$ ).

Ces trois conditions donnent un maximum local si la concavité de $\mathcal{L}$ est assurée (cf annexe A), ce qui est le cas pour des hypothèses raisonnables de fonctions de demande et de coûts.

Afin d'illustrer ces résultats, nous nous proposons de spécifier le modèle et d'expliciter les solutions pour commenter leurs évolutions par rapport aux paramètres pertinents du modèle. Soit une demande inverse de tickets émanent de la part de consommateurs : $p(N)=\alpha \bar{N}-\alpha N$. Le prix est nul lorsque la demande est maximale, i.e. égale à $\bar{N}$. La demande est décroissante avec le prix. Soit une demande d'espaces commerciaux, décroissante avec le prix de la location d'espace et croissante avec le nombre de passagers de la forme suivante $S(r, N)=\beta N-\rho r+\bar{S}$. Les coûts de la compagnie et de l'aéroport sont supposés linéairement croissants avec le nombre de passagers : $C V(N)=\gamma N$ et $C(N)=\theta N$. On suppose $\alpha>0, \beta>0, \rho>0, \gamma>0, \theta>0$, $\bar{N}>0, \bar{S}>0$. Les conditions de premier ordre donnent :

$$
\begin{array}{rlrl}
\frac{\partial \mathcal{L}}{\partial N}=-2 N^{*} \alpha+\bar{N} \alpha+r^{*} \beta-\gamma-\theta+\mu^{*}\left(\bar{a}+r^{*} \beta-\gamma\right) & & =0 & \left(C N_{1}\right) \\
\frac{\partial \mathcal{L}}{\partial r}=\left(1+\mu^{*}\right)\left(\bar{S}+N^{*} \beta-2 r^{*} \rho\right) & =0 & \left(C N_{2}\right) \\
\mu^{*}\left(\gamma N^{*}+C F-\bar{A}-r^{*}\left(\beta N^{*}-\rho r^{*}+\bar{S}\right)-\bar{a} N^{*}\right) & =0 & \left(C N_{3}\right),
\end{array}
$$

Le programme est concave sous des conditions raisonnables présentées en annexe A et le programme admet donc un maximum global. La caractérisation de ce maximum amène à discussion, notamment concernant la contrainte portant sur la redevance aéronautique maximale. Cette redevance aéronautique est un paramètre fixé par le régulateur et s'impose à la compagnie. $\bar{a}$ est un prix plafond fixé en fonction de règle de régulation qui tiennent compte des coûts incrémentaux des aéroports et des conditions de marché. Le cas qui va nous intéresser le plus est celui pour lequel ce prix plafond autorisé est suffisamment élevé pour ne pas devenir une contrainte pour la compagnie. En effet, cela laisse ainsi l'opportunité à la compagnie de choisir un niveau de redevance éventuellement plus faible que le prix plafond, voire éventuellement négatif (i.e. une subvention de l'aéroport en sa faveur) en fonction des paramètres du modèle (notamment des caractéristiques de la demande de service de transport).

Proposition 2 Si les consommateurs sont suffisamment sensibles au prix du billet $\alpha \geq \underline{\alpha}=$ $\frac{-\bar{S} \beta+2(\gamma+\theta) \rho}{2 N \rho}$, alors la compagnie aérienne peut obtenir du gestionnaire de l'infrastructure une charge d'accès strictement inférieure au plafond imposé par le régulateur $\left(\mu^{*}=0\right)$.

Par ailleurs, plus l'aide d'état maximale Ā sera élevée, plus la redevance aéronautique optimale $a^{*}$ sera faible. La redevance optimale revient même à une subvention en faveur de la LCC si le profit généré par l'activité commerciale est suffisamment élevé. 


\section{Preuve.}

Voir annexe B.

C'est la demande des passagers qui détermine in fine la stratégie optimale de la compagnie : si cette demande a une sensibilité élevée au prix du billet, alors le choix d'un prix faible aura beaucoup d'effet sur le nombre de passagers voyageant. Afin de pouvoir équilibrer son profit, la compagnie ajuste la redevance aéronautique a un niveau faible pour maintenir son profit car cela diminue de fait ses coûts ${ }^{14}$. La compagnie, grâce à son prix du billet peu élevé peut attirer plus de trafic sur l'aéroport et ainsi accroître le profit réalisé sur l'activité commerciale. Par ailleurs, plus l'externalité exercée par les passagers sur l'activité commerciale est importante (mesurée par le paramètre $\beta$ dans notre modèle) et plus le profit commercial sera élevé. À l'équilibre, plus l'impact de l'externalité est élevé, plus le nombre de passagers transportés à l'équilibre est important. Ainsi, le niveau retenu pour la redevance aéronautique peut conduire à subventionner la LCC dès lors que le profit commercial de l'aéroport est suffisamment important. Cette externalité est liée à la définition que nous avons retenue de la nature biface de l'activité aéroportuaire.

Il convient également de noter qu'il est possible d'établir une équivalence entre l'intensité de réduction obtenue par la LCC et la mesure de soutien $\bar{A}$. La compagnie peut exiger une ristourne d'autant plus forte qu'elle considère les gains potentiels liés à la convention pour l'aéroport et la jurisprudence concurrentielle européenne en matière d'encadrement des aides publiques. Elle peut négocier en position de force des réductions importantes de redevances qui lui permettront de s'accaparer une large part des gains et qui conduiront à des niveaux d'aides plus élevés. Une régulation des redevances par prix plafond ne permet pas de limiter le niveau de la baisse des redevances et donc de "plafonner" le montant de l'aide. Ce faisant, les deux instruments de réglementation ex ante et ex post sont liés. L'imposition d'un prix plancher par exemple, i.e. d'une valeur minimale prise par la redevance aéronautique, permettrait de diminuer le niveau de la mesure de soutien à l'infrastructure sans pour autant modifier les résultats de l'équilibre. Elle conduirait à la fois à limiter la capacité de la LCC à s'approprier les gains de l'échange et à réduire le niveau des fonds publics nécessaires au titre de l'aide. En d'autres termes, il peut être préférable de plafonner, au travers d'une régulation par prix plancher du montant des redevances aéronautiques, le montant des aides qui peuvent être versées par un gestionnaire d'aéroport dépourvu de pouvoir de marché.

Dans le cadre de notre modèle, il n'est pas possible directement de réaliser une analyse de bien-être puisque nous n'avons pas spécifié les objectifs de chacun des régulateurs. On peut, en revanche, approcher cette analyse en comparant les profits générés par le contrat avec l'investissement réalisé par le gestionnaire d'aéroport lorsqu'il demande l'aide d'état. Si l'opération dégage des ressources, on pourra alors considérer que l'investissement de la mesure de soutien de l'activité aura été rentable.

Il s'agit ainsi de comparer les profits de la compagnie aérienne et de l'aéroport à la valeur de

14. La compagnie ne peut pas récupérer via le contrat qu'elle offre le profit complet de l'aéroport. Sa meilleure option est donc de réduire la partie du profit de l'aéroport qui correspond aux coûts des services aéronautiques. 
l'aide d'état demandée à l'équilibre. D'après la proposition 1, l'aéroport ne fait aucun profit et l'aide demandée est maximale i.e. vaut $\bar{A}$. Il faut ainsi s'assurer que

$$
r^{*} S\left(r^{*}, N^{*}\right)+p\left(N^{*}\right) N^{*}-C\left(N^{*}\right)-C V\left(N^{*}\right)-C F+\bar{A} \geq \bar{A}
$$

D'après notre spécification, cela revient à dire que l'investissement est rentable dans la mesure où les coûts fixes ne sont pas trop importants. Cette condition est somme toute plutôt naturelle : si les coûts fixes sont très élevés, l'infrastructure, malgré un soutien important, ne sera jamais en mesure au travers de son activité de couvrir les coûts fixes. La borne supérieure pour les valeurs acceptables de coûts fixes contraint l'ensemble des paramètres acceptables. Il faut en effet prendre $\alpha>\alpha_{4}=\frac{5 \beta^{2}}{4 \rho}$, autrement dit une sensibilité de la demande de transport avec le prix du ticket encore plus importante que $\underline{\alpha}$.

Proposition 3 La mesure de soutien peut être considérée comme un investissement rentable au sens d'un investisseur privé dès lors que les coûts fixes ne sont pas excessifs. Le profit de la compagnie aérienne est en effet supérieur à l'aide publique, rendant l'investissement profitable lorsque

$$
C F \leq \overline{C F}=\frac{-\bar{S}^{2} \alpha\left(-5 \beta^{2}+4 \alpha \rho\right)+(-\bar{N} \alpha+\gamma+\theta)\left(\beta^{2}+12 \alpha \rho\right)(\bar{S} \beta-(-\bar{N} \alpha+\gamma+\theta) \rho)}{\left(\beta^{2}-4 \alpha \rho\right)^{2}}
$$

qui est vraie pour $\alpha \geq \operatorname{Max}\left[\underline{\alpha}, \frac{5 \beta^{2}}{4 \rho}\right]$.

Preuve.

Voir annexe C.

\section{Conclusion}

Notre modèle illustre la rationalité pour un gestionnaire d'infrastructure aéroportuaire se comportant comme un investisseur privé en économie de marché d'accorder des soutiens à l'exploitation en faveur des LCC, soutiens, qui plus est, pérennes. À ce titre, il conforte les assouplissements apportés par les lignes directrices de 2014 et justifierait a priori des ristournes sur les redevances aériennes qui ne seraient plus transitoires. La prise en compte des externalités entre les segments aéronautiques et non aéronautiques de l'activité des aéroports fait que des aides versées aux LCC pour générer et pérenniser des dessertes (i.e. pour augmenter les flux passagers) peuvent être "financées" par les revenus additionnels générés sur le volet commercial (parkings, boutiques, etc...). Ce faisant un investisseur privé en économie de marché pourrait accepter de ne pas couvrir l'ensemble de ses coûts sur ce versant en prenant en compte les gains induits sur le second. Des réductions drastiques des redevances (une quasi-gratuité) voire des clauses de partage des revenus commerciaux (en d'autres termes des redevances aéroportuaires négatives) peuvent donc faire l'objet d'une défense économique sur la base de l'efficience, même si elles sont pérennes et non transitoires. 
De telles conventions entre gestionnaires d'aéroports secondaire et compagnies aériennes permettent potentiellement pour le premier d'opérer à l'équilibre ou du moins de limiter le déficit d'exploitation. Un gain procède donc de l'échange. Il peut se décliner en deux volets. Un premier concerne les retombées pour le territoire (liées par exemple au désenclavement, au renforcement de la connectivité,...). Un second peut résider dans l'économie de ressources publiques nécessaires pour compenser le déficit d'exploitation de l'aéroport. Or, la LCC peut grâce à une éventuelle situation de monopsone (observée dans de nombreux aéroports régionaux européens) confisquer une grande part de ce surplus. Cela se traduit par une moindre économie de fonds publics pour la collectivité. Une réponse serait de plafonner l'intensité de l'aide. C'est ce que fait la Commission ex post au travers de sa pratique décisionnelle.

Une autre solution, que nous mettons en exergue dans notre travail, serait de tenir compte de l'équivalence entre intensité de la ristourne sur les taxes aéroportuaires et intensité de l'aide publique nécessaire. La régulation actuelle par prix plafond fait sens pour les aéroports dotés de pouvoir de marché vis-à-vis des compagnies aériennes. Elle permet d'éviter que ceux-ci n'abusent de leur situation pour extorquer une part excessive du surplus des opérateurs pour lesquels ces aéroports sont des facilités essentielles. Cette situation n'est pas de mise pour les aéroports régionaux. Ceux-ci sont en concurrence les uns avec les autres pour les LCC. La demande qui s'adresse à ces dernières, notamment pour le segment de clientèle loisirs, est très sensible au prix et moins aux localisations desservies. Les gestionnaires d'aéroports doivent eux garantir coûte que coûte l'existence de dessertes sur leurs infrastructures où ont été réalisés ex ante des investissements coûteux en investissements publics et non redéployables. Face à cette situation de dépendance économique aggravée par des sunk costs, la LCC peut mettre en oeuvre une stratégie de holp-up contractuel passant par l'exigence d'une fixation des redevances aéroportuaires très en deçà du prix plafond, voire d'une gratuité si ce n'est d'une redevance négative (passant par partage des revenus commerciaux, cofinancements de campagnes promotionnelles etc.).

Une régulation par prix plafond ne permet pas de limiter l'importance de l'aide nécessaire pour compenser le fait que la LCC s'approprie tout le gain lié à l'accord. Il faudrait sur le principe limiter le montant de l'aide accordée sous forme d'une réduction des redevances. Or, il ne s'agit pas toujours d'une aide publique si l'on considère les cas où les termes de l'accord satisfont aux critères du principe de l'investisseur privé. Il s'agit donc de trouver un moyen de limiter la capacité de la LCC de tirer profit de la dépendance de l'aéroport. Nous avons noté dans notre modèle que le gain qu'elle peut s'approprier est d'autant plus élevé que l'externalité exercée par les passagers sur l'activité commerciale est importante. Nous n'avons pas réalisé une analyse en termes de bien-être dans le cadre de cet article mais nous pouvons également considérer que le coût collectif de cette captation sera d'autant plus important que le coût marginal des fonds publics mobilisés sera important. Notre proposition est alors de plafonner la capacité de la LCC à s'accaparer ce gain en substituant pour les aéroports dépourvus de pouvoir de marché à une régulation ex ante des redevances aéroportuaires par prix plafonds, une régulation par prix plancher. Celle-ci permet de limiter ex ante l'intensité de l'aide. 


\section{A Concavité du programme de la compagnie aérienne}

Les conditions suffisantes à l'obtention d'un maximum s'il existe dépendent de la concavité de $\mathcal{L}$. Il est possible de décomposer ce lagrangien en différentes fonctions dont la concavité assure d'obtenir une solution optimale. On peut réécrire $\mathcal{L}$ de la façon suivante :

$\mathcal{L}=p(N) N-C(N)-a N+(1+\mu)(r S(r, N)-C V(N)+a N)+\mu(\bar{a}-a) N+(1+\mu)(\bar{A}-C F)$

La première expression correspond au profit de la compagnie aérienne : $p(N) N-C(N)-a N$. Ce profit est concave en $N>0$ ssi $\frac{\partial^{2} \Pi_{L C C}}{\partial N^{2}} \leq 0$, ce qui nous donne la condition suffisante $\left(C S_{L C C}\right)$

$$
\begin{array}{llll}
\frac{1}{N}\left(-2 \frac{\partial p}{\partial N}+\frac{\partial^{2} C}{\partial N^{2}}\right) & \geq & \frac{\partial^{2} p}{\partial N^{2}} & \left(C S_{L C C}\right) \\
\text { pour } & N>0
\end{array}
$$

On s'assurera que $N \neq 0$ à l'équilibre. Cette condition est typiquement satisfaite si la demande des passagers $p(N)$ est linéaire en $N$. Sinon, la condition requiert que si les effets du prix sur le nombre de passagers au second ordre sont croissants, i.e. $\frac{\partial^{2} p}{\partial N^{2}}>0$, ces derniers restent limités par rapport aux effets de demande de premier ordre, ajoutés aux effets de la convexité des coûts de la compagnie hypothèse de travail du modèle).

La seconde expression correspond au profit de l'aéroport, multiplié par le multiplicateur $\mu \geq 0:(1+\mu)(r S(r, N)-C V(N)+a N)$.

De même que pour la compagnie, la concavité de ce programme est souhaitable pour l'intérêt de l'analyse. Elle est assurée du moment que

$$
\begin{array}{llll}
\frac{\partial^{2} C V}{\partial N^{2}} & \geq & r \frac{\partial^{2} S}{\partial N^{2}} & \left(C S_{\text {aéroport }}^{1}\right) \\
\frac{-2}{r}\left(\frac{\partial S}{\partial r}\right) & \geq & \frac{\partial^{2} S}{\partial r^{2}} & \left(C S_{\text {aéroport }}^{2}\right) \\
\left(r \frac{\partial^{2} S}{\partial N^{2}}-\frac{\partial^{2} C V}{\partial N^{2}}\right)\left(r \frac{\partial^{2} S}{\partial r^{2}}+2 \frac{\partial S}{\partial r}\right)-\left(\frac{\partial S}{\partial N}\right)^{2} & & & \left(C S_{\text {aéroport }}^{3}\right) \\
\text { pour } & r>0 &
\end{array}
$$

On s'assurera que $r \neq 0$ à l'équilibre. $\left(C S_{\text {aéroport }}^{1}\right)$ est assurée car les coûts variables de l'aéroport sont convexes, et l'impact de l'externalité sur la demande de location d'espaces est décroissant avec le nombre de passagers présents dans l'aéroport. Les rendements de l'externalité positive sont supposés en effet décroissant par souci de réalisme. ( $\left.C S_{\text {aéroport }}^{1}\right)$ est typiquement vérifiée pour une demande d'espaces $S(r, N)$ linéaire en le prix de location $r$. Enfin, $\left(C S_{\text {aéroport }}^{3}\right)$ est une condition à vérifier. Elle est valide du moment que l'effet de l'externalité sur la demande de location d'espaces n'est pas trop fort par rapport aux effets sur les coûts et l'effet direct du prix $r$ sur la demande $S(r, N)$. Enfin, la dernière expression $\mu(\bar{a}-a) N+(1+\mu)(\bar{A}-C F)$ est linéaire en $N$, donc ne modifie pas la concavité du programme général.

En conclusion, si les conditions $\left(C S_{L C C}\right),\left(C S_{\text {aéroport }}^{2}\right)$ et $\left(C S_{\text {aéroport }}^{3}\right)$ sont satisfaites, alors c'est suffisant à obtenir un maximum local. Si elle sont strictement satisfaites, le maximum sera 
global. Dans le cas de notre spécification, les conditions sont les suivantes :

$$
\begin{array}{llll}
\frac{\partial^{2} \mathcal{L}}{\partial N^{2}} & = & -2 \alpha & \left(C S_{1}\right) \\
\frac{\partial^{2} \mathcal{L}}{\partial r^{2}} & = & -2 \rho(1+\mu) & \left(C S_{2}\right) \\
\frac{\partial^{2} \mathcal{L}}{\partial N^{2}} \frac{\partial^{2} \mathcal{L}}{\partial r^{2}} & -\left[\frac{\partial^{2} \mathcal{L}}{\partial r \partial N}\right]^{2} & =-\beta^{2}(1+\mu)-4 \alpha \rho & \left(C S_{3}\right) \\
\text { pour } & \mu \geq 0 & &
\end{array}
$$

$\left(C S_{1}\right)<0$ et $\left(C S_{2}\right)<0$ pour tout $\alpha>0$ et $\rho>0$ et $\mu \geq 0 . C S_{3}>0$ ssi $\alpha \geq \alpha_{0}=\frac{(1+\mu) \beta^{2}}{4 \rho}$

\section{B Preuve proposition 2:}

Les conditions de premier ordre donnent:

$$
\begin{array}{lll}
\frac{\partial \mathcal{L}}{\partial N}=0 & \Leftrightarrow & N^{*}=\frac{\bar{S} \beta\left(1+\mu^{*}\right)+2 \rho\left(\bar{N} \alpha+\gamma+\theta-\bar{a} \mu^{*}+\gamma \mu^{*}\right)}{4 \rho \alpha-\beta^{2}\left(1+\mu^{*}\right)} \\
\frac{\partial \mathcal{L}}{\partial r}=0 & \Leftrightarrow & r^{*}=\frac{2 \bar{S} \alpha+\beta\left(\bar{N} \alpha-\gamma\left(1+\mu^{*}\right)-\theta+\bar{a} \mu^{*}\right)}{4 \rho \alpha-\beta^{2}\left(1+\mu^{*}\right)} \\
\mu^{*}\left(\gamma N^{*}+C F-\bar{A}-r^{*}\left(\beta N^{*}-\rho r^{*}+\bar{S}\right)-\bar{a} N^{*}\right) & & =0 \\
\operatorname{pour} & \mu^{*} \geq 0 &
\end{array}
$$

Un certain nombre de contraintes sont à vérifier pour calibrer le modèle correctement. Plus spécifiquement, nous devons nous assurer que $N^{*} \geq 0, N^{*} \leq \bar{N}, r^{*} \geq 0$. Deux cas sont à envisager, selon que la contrainte est saturée ou non. Supposons que $\mu^{*}=0$

$-N^{*} \geq 0$ ssi $\alpha \geq \alpha_{1}=\frac{-\bar{S} \beta+2 \rho(\gamma+\theta)}{2 \bar{N} \rho}$; avec $\alpha_{1}>0$ si $-\bar{S} \beta+2 \rho(\gamma+\theta)>0$.

$-N^{*} \leq \bar{N}$ ssi $\alpha \geq \alpha_{2}=\frac{-\bar{S} \beta+2 \rho(\gamma+\theta)+\bar{N} \beta^{2}}{6 \bar{N} \rho}, \alpha_{2}>0$ si $\alpha_{1}>0$.

$-r^{*} \geq 0$ ssi $\alpha \geq \alpha_{3}=\frac{\beta(\gamma+\theta)}{N \beta+2 \bar{S}}$, avec $\alpha_{3}>0$ sans condition supplémentaire.

Les contraintes imposent toutes d'avoir un $\alpha$ minimum, elles sont donc toutes compatibles entre elles. Il reste maintenant à savoir quelle est la borne inférieure pour $\alpha$ pertinente. Comparons $\alpha_{2}$ et $\alpha_{0}$. Le signe de leur différence $\alpha_{2}-\alpha_{0}$ dépend du signe de $\left(-2 \bar{S} \beta+\bar{N} \beta^{2}+4(\gamma+\theta) \rho\right)$ laquelle différence peut être décomposée en deux sous éléments, $\left.-\bar{S} \beta+\bar{N} \beta^{2}+2(\gamma+\theta) \rho\right)>0$ car $\alpha_{2}>0$ et $-\bar{S} \beta+2(\gamma+\theta) \rho)>0$ si $\alpha_{1}>0$. Donc le signe de la différence est positive, i.e. $\alpha_{2}>\alpha_{0}$. De même, le signe de la différence entre $\alpha_{3}$ et $\alpha_{0}$ dépend de la même condition et est donc vrai pour l'ensemble des paramètres $\alpha$ admissibles. La comparaison des seuils $\alpha_{3}$ et $\alpha_{1}$ de même que des seuils $\alpha_{2}$ et $\alpha_{1}$ donne le même résultat : $\alpha_{1}>\alpha_{3}$ et $\alpha_{1}>\alpha_{2}$ sous la condition que $\alpha_{1}>0$ et $\alpha_{2}>0$. Il n'est donc pas nécessaire d'imposer une condition supplémentaire pour classer $\alpha_{2}$ et $\alpha_{3}$. On définit $\underline{\alpha}=\alpha_{1}$.

La contrainte sur la redevance aéronautique n'est pas saturée si le niveau plafond fixé par le régulateur est suffisamment haut. On ne peut complètement répondre à cette question sans fixer plus avant les paramètres. Cependant, il existe un ensemble non nul de paramètres pour lesquels $a^{*}<\bar{a}$. Ces paramètres satisfont la contrainte suffisante à l'optimum :

$$
\frac{-\bar{A}+C F+C V\left(N^{*}\right)-r^{*} S\left(r^{*}, N^{*}\right)}{N^{*}}<\bar{a}
$$

Si les paramètres satisfont cette contrainte, alors il n'est pas nécessaire d'envisager le cas $\mu^{*}>0$. 
La solution pour la redevance aéronautique est alors fonction décroissante de $\bar{A}$, comme le montre le signe de la dérivée première de $a^{*}$ par rapport à $\bar{A}$. Après calcul, le signe de la dérivée dépend du signe de l'expression suivante $\bar{S} \beta+2 \rho(\bar{N} \alpha-\gamma-\theta)$. Ce signe est négatif pour tout $\alpha \geq \underline{\alpha}$.

La dérivée de $N^{*}$ par rapport au paramètre d'externalité $\beta$ est positive du moment que $\alpha>\alpha_{4}=\frac{\bar{S}\left(2 \bar{S} \beta+\bar{N} \beta^{2}-4(\gamma+\theta) \rho\right.}{4 N \rho(S+N \beta)}$. Ceci ne rajoute pas de contrainte puisque le signe de la différence de $\alpha_{4}$ et $\underline{\alpha}$ dépend du signe de $\left(-2 \bar{S} \beta+\bar{N} \beta^{2}+4(\gamma+\theta) \rho\right)$ dont on a déjà démontré plus haut la négativité. Pour tout $\alpha>\underline{\alpha}, \frac{\partial N^{*}}{\partial \beta}>0$.

\section{Preuve proposition 3 :}

Afin de vérifier la profitabilité de l'opération pour l'investisseur public, il faut vérifier que le profit de la compagnie augmenté de celui de l'aéroport est à l'optimum supérieur à $\bar{A}$, l'aide versée. Il faut alors vérifier que

$$
r^{*} S\left(r^{*}, N^{*}\right)+p\left(N^{*}\right) N^{*}-C\left(N^{*}\right)-C V\left(N^{*}\right)-C F \geq 0
$$

Après calculs dans le cadre de notre spécification, la condition dépend du signe de l'expression suivante :

$$
-C F\left(\beta^{2}-4 \alpha \rho\right)-\bar{S}^{2} \alpha\left(-5 \beta^{2}+4 \alpha \rho\right)+(-\bar{N} \alpha+\gamma+\theta)\left(\beta^{2}+12 \alpha \rho\right)(\bar{S} \beta-(-\bar{N} \alpha+\gamma+\theta) \rho) \geq 0
$$

Avant toute chose, il faut s'assurer que l'expression n'est pas négative pour tous les paramètres possibles. La première partie représente les coûts fixes et est négative. La deuxième partie dépend de la valeur des paramètres et enfin, le troisième terme est de valeur positive pour $\alpha \in[\underline{\alpha}, \bar{\alpha}]$. On est donc assuré de trouver un sous-ensemble de paramètres qui satisfont cette inégalité strictement. Une condition pourrait être imposée par exemple sur la valeur maximale que peuvent prendre les coûts fixes.

$$
C F \leq \overline{C F}=\frac{-\bar{S}^{2} \alpha\left(-5 \beta^{2}+4 \alpha \rho\right)+(-\bar{N} \alpha+\gamma+\theta)\left(\beta^{2}+12 \alpha \rho\right)(\bar{S} \beta-(-\bar{N} \alpha+\gamma+\theta) \rho)}{\left(\beta^{2}-4 \alpha \rho\right)^{2}}
$$

Ce seuil $\overline{C F}$ est positif ssi

$$
-\bar{S}^{2} \alpha\left(-5 \beta^{2}+4 \alpha \rho\right)+(-\bar{N} \alpha+\gamma+\theta)\left(\beta^{2}+12 \alpha \rho\right)(\bar{S} \beta-(-\bar{N} \alpha+\gamma+\theta) \rho)>0 .
$$

Cette condition est vraie à la condition suffisante suivante $\alpha>\alpha_{4}=\frac{5 \beta^{2}}{4 \rho}$. 


\section{Références}

[1] Aéroports De Paris [2010-2015], Financial report.

[2] Armstrong M. [2006] , "Competition in two-sided markets », RAND Journal of Economics, 37, p. 668-691.

[3] Commission Européenne [2005], Community Guidelines on Financing of Airports and Start-up Aid to Airlines Departing from Regional Airports.

[4] Commission Européenne [2014], Guidelines on State aid to airports and airlines, Journal Officiel UE, volume 57, C99, 4 avril, p. 3-34.

[5] Conseil SupéRieur de l'Aviation Civile [2017], Rapport sur le maillage aéroportuaire français, Commissariat Général à l'Egalité des Territoires et Direction Générale de l'Aviation Civile.

[6] European Court of Advisors [2014], «EU-funded airports infrastructures : poor valuefor-money », Special Report, vol. 21, Luxembourg, 72p.

[7] Evans, D. et Schmalensee R. [2007], Catalyst Code, Havard Business School Press.

[8] Fröhlich K. [2011], « Airports as Two-Sided Markets? A Critical Contribution », Working Paper, University of Applied Sciences, Bremen.

[9] Fu X., Homsombat W. et Oum T-H. [2011], « Airport-Airline Vertical Relationships, Their Effects and Regulatory Policy Implications », Journal of Air Transport Management, vol.17, p. 347-53.

[10] Fu X. et Zhang A. [2010], « Effects of Airport Concession Revenue Sharing on Competition and Social Welfare », Journal of Tansport Economics and Policy, 44, Part 2, p. 119-138.

[11] Gillen D. [2011], « The Evolution of Airport Ownership and Governance », Journal of Air Transport Management, 17(1), pp.3-13.

[12] Hagiu, A et Wright J. [2015], « Multi-sided platforms », International Journal of Industrial Organization, 43, p. 162-174.

[13] International Transport Forum round tables, 2009, Competitive interaction between airports, airlines and high speed train, OECD.

[14] Ivaldi M., Sokullu S. et Toru T. [2015], « Airport Prices in a Two-Sided Market setting: Major US Airports », Working Paper TSE, 587, 37p.

[15] Malavolti E. [2016], « Single Till or Dual Till at airports : a two-sided market analysis », Transportation Research Procedia, 14, p. 3696-3703.

[16] Malavolti E. et Marty F. [2010], « Analyse économique des aides publiques versées par les aéroports régionaux aux compagnies low cost », Revue Européenne de Droit de la Consommation / European Journal of Consumer Law, 2010/3-4, septembre, p. 529-558.

[17] Malavolti E. et Marty F. [2013], « La gratuité peut-elle avoir des effets anticoncurrentiels? Une perspective d'économie industrielle sur le cas Google», dans Martial-Braz N. et 
Zolynski C., (s.d.), La gratuité un concept aux frontières de l'économie et du droit, Collection Droit et Economie, LGDJ, Paris, p.71-89.

[18] Malina R., Albers S. et Kroll N. [2012], « Aiport Incentive Programs? A European Perspective »,Transport Reviews , 32(4), p. 1-19.

[19] Perrot A. [2014], Séminaire Philippe Nasse, French Competition Authority, "Problèmes de concurrence liés au fonctionnement des aéroports Approche économique".

[20] REY P. [2003], « Economics of vertical restraints », dans Economics for an Imperfect World: Essays in Honor of Joseph E. Stiglitz, Richard Arnott, Bruce Greenwald, Ravi Kanbur, and Barry Nalebuff (eds.), Cambridge : MIT Press, chapter 14, p. 247-268.

[21] Rochet, J-C et Tirole J. [2003], « Platform Competition in Two-Sided Markets », Journal of European Economic Association, 1(4), p. 990-1029.

[22] Rochet J-C. et Tirole J. [2006], « Two-Sided Markets : A Progress Report », Rand Journal of Economics, The RAND Corporation, 37, p. 645-667.

[23] StaRkIE D. [2001], « Reforming UK airport regulation», Journal of Transport Economics and Policy, 35(1), p. 199-135.

[24] Starkie D. et Yarrow G. [2008], report for the CAA UK, "The Single-Till Approach to the Price Regulation of Airports".

[25] Torres, E., Dominguez, J.S., Valdès, L. et Aza R. [2005], « Passenger waiting time in an airport and expenditure carried out in the commercial area », Journal of Air Transport Management, 11, issue 6, p. 363-367.

[26] Verdier M. [2016], « Les développements récents de la littérature sur les plates-formes » , Revue Economique, 67, p. 25-38.

[27] Wright J. [2007], « Slotting contracts and consumers welfare », Antitrust Law Journal, 742), p. 439-473. 